\title{
Manejo práctico del derrame pleural
}

\author{
J. M. PORCEL-PÉREZ \\ Servicio de Medicina Interna. Hospital Universitario Arnau de Vilanova. Lleida
}

\author{
PRACTICAL MANAGEMENT OF PLEURAL EFFUSION
}

\section{RESUMEN}

Existen numerosas enfermedades que se pueden asociar con derrame pleural. Cuando se descubre un derrame pleural, se debe dar respuesta a dos preguntas: 1) ¿es el derrame un trasudado o un exudado? y 2) si el derrame es un exudado, ¿cuál es la enfermedad que lo ha producido?. El análisis del líquido pleural mediante toracocentesis permite establecer un diagnóstico definitivo en más de dos terceras partes de los pacientes. El resto pueden requerir un período de observación en espera de la resolución espontánea o el empleo de métodos diagnósticos no invasivos (técnicas de imagen) o invasivos (broncoscopia, biopsia pleural, toracoscopia).

PALABRAS CLAVE: Derrame pleural. Derrame pleural maligno. Derrame pleural paraneumónico. Tuberculosis.

\begin{abstract}
There are many different diseases that can be associated with pleu ral effusions. When a pleural effusion is discovered, two questions need to be answered: 1) is the effusion a transudate or is it an exudate?, and 2) if the effusion is an exudate, what is the disease responsible for its pro duction?. Answers to these questions can be obtained in more than twothirds of patients testing the pleural fluid by diagnostic thoracentesis. The remainder may require watchful waiting until resolution or further diagnostic procedures, either non-invasive (radiologic imaging) or inva sive (bronchoscopy, pleural biopsy, thoracoscopy).
\end{abstract}

KEY WORDS: Pleural effusion. malignant pleural effusion. parapneu monic pleural effusion. tuberculosis.

Porcel Pérez JM. Manejo práctico del derrame pleural. An Med Interna (Madrid) 2002; 19: 202-208.

\section{INTRODUCCIÓN}

El derrame pleural (DP) se desarrolla cuando la formación de líquido pleural (LP) excede su absorción (1). Normalmente se genera alrededor de $0,01 \mathrm{ml} / \mathrm{kg}$ cada hora de LP a partir de los capilares de la pleura parietal. Sin embargo, en estados patológicos la formación de LP aumenta extraordinariamente. El origen del LP en numerosas enfermedades es el espacio intersticial del pulmón. Aproximadamente el $20 \%$ del líquido que entra en el espacio intersticial del pulmón sale de este órgano a través de la cavidad pleural. El LP que se forma durante la insuficiencia cardiaca izquierda, la neumonía o el embolismo pulmonar probablemente se origina en el espacio intersticial pulmonar. En otras enfermedades, el LP se genera en los capilares de la pleura visceral o parietal o en la cavidad peritoneal. Obviamente, en el hemotórax el líquido procede de un vaso sanguíneo mientras que en el quilotórax lo hace directamente del conducto torácico.

El LP se elimina de la cavidad pleural a través de los linfáticos de la pleura parietal, que son capaces de absorber al menos $0,20 \mathrm{ml} / \mathrm{kg} / \mathrm{h}$. Por lo tanto, si los linfáticos son normales, el grado de formación de LP debe aumentar 20 veces por encima de lo normal para superar la capacidad que tienen aquéllos para eliminarlo. En la tabla I se citan las causas que aumentan la formación o disminuyen la absorción de LP. Cuando se observa un DP, nuestros esfuerzos se deben dirigir a determinar cuál de los factores referidos en la tabla I es responsable de la acumulación de LP.

\section{DIFERENCIACIÓN ENTRE TRASUDADOS Y EXUDADOS}

Los DP se han clasificado clásicamente en trasudados y exudados. Los trasudados son ultrafiltrados del plasma en la pleura que se forman porque se alteran las presiones hidrostáticas $u$ oncóticas sistémicas que influyen sobre la formación o absorción de LP. El líquido se puede originar en el pulmón (insuficiencia cardíaca) o, menos frecuentemente en la cavidad peritoneal (cirrosis). Por el contrario, el DP exudativo se desarrolla cuando se afectan las superficies pleurales o se incrementa la permeabilidad capilar local por una inflamación pleural o pulmonar, o bien se reduce el drenaje linfático del espacio pleural. Infecciones y neoplasias constituyen las etiologías principales de este tipo de derrames. La importancia de diferenciar entre

Trabajo aceptado: 7 de julio de 2001 


\section{TABLA}

\section{ECANISM OS DE FO RM ACIÓ N DEL DERRAM E PLEURAL}

Aumento en la formación de líquido pleural

Aumento del líquido intersticial pulmonar (IC, neumonía, embolia pulmonar)

Aumento de la presión intravascular en la pleura (IC, obstrucción venosa)

Aumento de permeabilidad de capilares pleurales (neumonía, neoplasia)

Disminución de la presión oncótica sanguínea (hipoalbuminemia)

Disminución de la presión pleural (atelectasia)

Aumento de líquido en la cavidad peritoneal (hidrotórax hepático)

Rotura del conducto torácico (quilotórax)

Rotura de vasos sanguíneos (hemotórax)

Disminución de la absorción de líquido pleural

O bstrucción de linfáticos que drenan la pleura parietal (neoplasia)

Aumento de las presiones vasculares sistémicas (IC, obstrucción venosa)

IC: insuficiencia cardiaca.

exudados y trasudados estriba en la necesidad de procedimientos diagnósticos ulteriores en el primer caso para conocer la etiología precisa, frente a la simple aplicación de un tratamiento en el segundo (p. ej.: diuréticos).

Desde su descripción hace 30 años, siguen vigentes los criterios de Light para diferenciar entre trasudados y exudados pleurales (2), según los cuales un exudado se define por la presencia de alguna de las siguientes características: a) cociente de proteínas entre LP y suero superior a $0,5,2$ ) cociente de lactato deshidrogenasa (LDH) entre LP y suero superior a 0,6 o 3) LDH del LP mayor que los dos tercios del límite superior de la LDH sérica (actualmente $307 \mathrm{UI} / 1$ en nuestro hospital). Estos criterios tienen una sensibilidad del 95-100\% y una especificidad del 75$80 \%$ para identificar exudados $(3,4)$. En dos estudios recientes $(5,6)$ se ha comprobado que la eliminación de uno de los elementos de la tríada (cociente LDH pleura/suero) no supone una merma en la eficacia discriminatoria (criterios de Light "abreviados"). Al priorizar la sensibilidad sobre la especificidad, los criterios de Light clasifican algunos trasudados como exudados, lo cual puede llevar a procedimientos diagnósticos innecesarios. Esto ocurre sobre todo en pacientes con insuficiencia cardíaca que están tomando diuréticos. Para resolver este problema, algunos autores $(7,8)$ recomiendan medir el gradiente de albúmina o de proteínas entre suero y LP, siempre que la sospecha clínica de trasudado no se vea apoyada por la aplicación de los criterios de Light. Cuando esta diferencia sea superior a 1,2 g/dl o 3,1 g/dl respectivamente, el paciente tendrá con toda probabilidad un trasudado.

Otro inconveniente de los criterios de Light es que se requiere una extracción simultánea de sangre para calcular los cocientes. No obstante, algunos estudios $(5,9,10)$ han demostrado que la combinación de LDH y colesterol en LP tiene un poder discriminante similar a los criterios de Light, con la ventaja de evitar la muestra sanguínea. De este modo, se considera que un LP es exudativo si tiene una concentración de colesterol superior a 60 $\mathrm{mg} / \mathrm{dl}$ o una LDH mayor de 307 U/l (dos tercios del límite superior de la normalidad para la LDH sérica) $(5,10)$.

\section{TORACOCENTESIS DIAGNÓSTICA}

Prácticamente todos los pacientes con DP deben someterse a una toracocentesis diagnóstica, excepto si existe muy escasa cantidad de LP $(<1 \mathrm{~cm}$ de espesor en la radiografía realizada en decúbito ipsilateral) o nos hallamos ante un cuadro clínico característico de insuficiencia cardíaca. El análisis del LP obtenido mediante esta sencilla técnica nos permite establecer una gran variedad de diagnósticos (Tabla II). En todos los LP es obligatorio el análisis de la concentración de proteínas, LDH o, eventualmente colesterol para clasificarlos como trasudados o exudados. Si los resultados orientan hacia esta última posibilidad, se deberán ampliar los estudios bioquímicos (recuento celular diferencial, $\mathrm{pH}$, glucosa, adenosin desaminasa -ADA-) y efectuar análisis microbiológicos (Gram, cultivos bacterianos y micobacterianos) y citológicos del LP en

TABLA II

DIAGNÓSTICOS QUE PUEDEN ESTABLECERSE M EDIANTE EL ANÁLISIS DE LÍQ UIDO PLEURAL

\begin{tabular}{ll}
\hline Enfermedad & Pruebas diagnósticas en LP \\
\hline Trasudado & Criterios de Light, colesterol $>45-60 \mathrm{mg} / \mathrm{dl}$ \\
Empiema & O bservación (pus, olor pútrido) \\
DPP complicado & $\mathrm{pH}<7$, glucosa $<40 \mathrm{mg} / \mathrm{dl}, \mathrm{Gram}$ o cultivo + \\
M alignidad & Citología positiva \\
Tuberculosis & Tinción o cultivo +, ADA $>40$ UI/I \\
DP pancreático & Isoenzima pancreática de la amilasa elevada \\
Perforación esofágica & Células epiteliales escamosas, partículas alimentarias, \\
& isoenzima salival de amilasa elevada \\
Artritis reumatoide & Citología característica \\
Lupus eritematoso sistémico & Células LE \\
Hemotórax & Hematócrito LP/S $>0,5$ \\
Quilotórax & Triglicéridos $>110$ mg/dl, quilomicrones \\
Urinotórax & Creatinina LP/S $>1$ \\
Diálisis peritoneal & Proteínas $<1 \mathrm{~g} / \mathrm{dl}$ y glucosa $300-400$ mg/dl \\
M igración extravascular de catéter & LP con características del líquido infundido
\end{tabular}

LP: líquido pleural; DPP: derrame pleural paraneumónico; ADA: adenosin desaminasa; DP: derrame pleural; LP/S: cociente entre líquido pleural y suero. 
cuestión. Aunque existen razones de coste-efectividad para defender este protocolo de actuación (11), en la práctica el juicio clínico debe prevalecer para decidir si de entrada se solicitan todos los estudios aludidos en el LP $(12,13)$. No está indicada la práctica rutinaria de una radiografía de tórax después de la toracocentesis diagnóstica si el paciente permanece asintomático (14).

\section{APARIENCIA DEL LÍQUIDO PLEURAL}

La apariencia macroscópica del LP puede ofrecer información de interés diagnóstico. Cuando el LP parece sangre, debemos determinar su hematócrito. Con frecuencia éste será mucho menor del esperado por la apariencia observada. Si el hematócrito del LP es mayor que el 50\% del existente en sangre periférica, el paciente tiene un hemotórax. La mayoría de los hemotórax se deben a traumatismos torácicos y menos frecuentemente son iatrógenos (p.ej. cateterización de venas centrales, colocación de tubos de toracostomía) o espontáneos (neoplasias, coagulopatías). En los demás casos, la apariencia hemática del LP tiene poca significación, aunque sugiere tres diagnósticos: neoplasia, embolia pulmonar o traumatismo.

Cuando el LP es turbio o lechoso, examinaremos el sobrenadante que resulta de su centrifugación. Si el sobrenadante es claro, la turbidez se debe a células o detritos, como ocurre en los DP infecciosos. Por el contrario, la causa de que persista la turbidez después de la centrifugación, es un alto contenido de lípidos. En estas circunstancias, el paciente tiene un quilotórax o un pseudoquilotórax. El diagnóstico de quilotórax se confirma por el hallazgo de unas concentraciones de triglicéridos en LP superiores a $110 \mathrm{mg} / \mathrm{dl}$ o de quilomicrones (15). El linfoma es la causa más frecuente de quilotórax.

\section{ANÁLISIS DEL LÍQUIDO PLEURAL}

Debemos obtener un recuento diferencial de leucocitos del LP en todos los exudados. Cuando predominan los polimorfonucleares $(>50 \%)$, el paciente tiene un proceso agudo que afecta a las superficies pleurales. Si existen infiltrados pulmonares concomitantes, el diagnóstico más probable es el derrame pleural paraneumónico (DPP), aunque debe considerarse la embolia pulmonar y el cáncer de pulmón. Si no hay infiltrados parenquimatosos, se valorará la posibilidad de una embolia pulmonar, procesos abdominales (pancreatitis, peritonitis), pleuritis viral o tuberculosa agudas y malignidad. Por el contrario, si predominan las células mononucleares en el LP (>50\% linfocitos), el paciente padece un proceso crónico que afecta a la pleura. Los diagnósticos más probables son la neoplasia y la tuberculosis y, menos frecuentemente, la embolia pulmonar, pleuritis viral en resolución o DP secundario a cirugía de bypass coronario. La eosinofilia pleural (>10\% eosinófilos), se relaciona con la presencia de sangre o aire en el espacio pleural. Tiene poco valor en el diagnóstico diferencial $(16,17)$ ya que también se observa en numerosas etiologías (DP infecciosos, malignos o idiopáticos).

La concentración pleural de LDH es un indicador fiable del grado de inflamación pleural. $\mathrm{Si}$, con las toracocentesis repetidas, se incrementa la cifra de LDH en LP, el grado de inflamación en el espacio pleural está progresando y debemos ser diligentes para establecer un diagnóstico definitivo (1).
La detección de unos niveles de glucosa bajos en LP $(<60$ $\mathrm{mg} / \mathrm{dl}$ ) indica que el paciente tiene probablemente uno de los siguientes trastornos: DPP complicado, neoplasia, tuberculosis o pleuritis reumatoide. Suele existir un paralelismo entre el descenso de la glucosa, la disminución del pH y la elevación de la LDH en el LP. La medición del pH del LP es muy útil en los DPP ya que una acidosis pleural significativa $(\mathrm{pH}<7)$ obliga a utilizar procedimientos invasivos (tubo de toracostomía) para resolver la infección pleural. El $\mathrm{pH}$ se mide en un aparato de gasometría arterial, previa recogida anaeróbica del LP en un tubo heparinizado. Contrariamente a la práctica médica de exigir la medición del $\mathrm{pH}$ pleural a los pocos minutos de la toracocentesis, se ha comprobado que sus valores no cambian significativamente a temperatura ambiente durante la primera hora de la extracción (18). Por consiguiente, la medición se puede efectuar durante dicho período de tiempo y sin necesidad de preservar la muestra en hielo. Se han descrito falsos descensos de $\mathrm{pH}$, no asociados con niveles bajos de glucosa, cuando se introduce anestesia en la cavidad pleural durante una toracocentesis, especialmente si el volumen del DP es pequeño (19).

Unos niveles de amilasa en LP por encima del límite superior de la amilasa sérica indican que el DP probablemente se debe a enfermedad pancreática, rotura esofágica o neoplasia. En el primer supuesto, el incremento depende de la isoenzima pancreática y en los dos restantes de la isoenzima salival.

\section{DIAGNÓSTICO DE LA PLEURITIS TUBERCULOSA}

Al ser el DP una forma paucibacilar de la infección tuberculosa, los estudios microbiológicos ofrecen un bajo rendimiento diagnóstico. Los cultivos del LP son positivos en menos de la tercera parte de los pacientes. La biopsia pleural con aguja, combinando el cultivo y la demostración de granulomas en la pieza, aumenta la eficacia diagnóstica al 90\% de casos (20). Sin embargo, es un procedimiento invasivo, sometido a errores de muestreo y, que se ha visto superado en los últimos 15 años por la fácil y barata determinación de ADA en el LP, en las áreas con alta prevalencia de tuberculosis.

En series españolas, puntos de corte de ADA pleural superiores a 40-45 UI/l tienen una sensibilidad cercana al 100\% y una especificidad del $90 \%$ para definir la naturaleza tuberculosa de un DP (20,21). Se observan falsos positivos en los DPP, empiemas, pleuritis reumatoide (fácilmente distinguibles clínicamente del DP tuberculoso) y, en menor medida, en neoplasias hematológicas y sólidas. En dos estudios recientes $(22,23)$ se ha observado menos de un $4 \%$ de falsas elevaciones de ADA entre los exudados linfocitarios. La ADA en la pleuritis tuberculosa aumenta a expensas de la isoenzima ADA-2 que se genera por la activación de los monocitosmacrófagos. Tanto la determinación de ADA-2 (>40 UI/l) como del cociente ADA1/ADA $(<0,42)$ en LP tienen también una alta rentabilidad diagnóstica $(20,24)$. En la práctica clínica, cuando un paciente joven ( $<40$ años) presenta un exudado con claro predominio linfocitario $(>80 \%)$, ADA pleural superior a $40 \mathrm{UI} / 1$ y citología del LP negativa para células malignas, se asume el diagnóstico de tuberculosis pleural y se inicia tratamiento antituberculoso (25).

La medición de interferón gamma, una linfocina liberada por los linfocitos T CD4+, en el LP presenta una sensibilidad $\mathrm{y}$ especifidad superiores al $95 \%$ para identificar pleuritis 
tuberculosa (26,27). El principal inconveniente es su carestía. La rentabilidad de la reacción en cadena de la polimerasa (PCR) para detectar la presencia de DNA de Mycobacterium tuberculosis en LP guarda íntima relación con la carga bacilar de la muestra, obteniéndose un bajo porcentaje de positividades cuando el cultivo del LP es negativo (28-30). En la práctica clínica, la sencilla determinación de ADA no ha podido ser desplazada por el interferón gamma, la PCR ni otros parámetros bioquímicos igualmente eficaces como el complejo terminal del complemento (31) o el receptor soluble de la interleucina-2 (32).

\section{DIAGNÓSTICO DEL DERRAME PLEURAL MALIGNO}

El examen citológico del LP es la forma menos invasiva, rápida y eficaz de establecer el diagnóstico de malignidad. Sin embargo, el porcentaje de DP malignos que se diagnostican con la citología oscila entre el 40 y el $87 \%$ (media del $60 \%$ ) (33). Existen varios factores que influyen en los resultados. En primer lugar, algunos tumores como los adenocarcinomas presentan con más frecuencia citologías positivas que otros tipos como el carcinoma escamoso, la enfermedad de Hodgkin, los sarcomas o el mesotelioma. En este último, el examen citológico es diagnóstico en menos del $20 \%$ de los casos. En segundo lugar, los DP paramalignos, es decir, aquellos que no derivan de la afectación neoplásica de la pleura, pero tienen relación con el tumor primario, dan resultados negativos en la citología. Algunos ejemplos son la neumonitis obstructiva que produce un DPP, la obstrucción del conducto torácico con la formación de un quilotórax, la embolia pulmonar o los trasudados secundarios a atelectasia obstructiva, hipoalbuminemia, síndrome de vena cava superior o pulmón atrapado. Finalmente, el rendimiento de la citología depende del número de muestras remitidas y de la experiencia del citólogo. Si se envían tres especímenes separados de LP a un citólogo experimentado, se puede obtener un diagnóstico positivo en cerca del $80 \%$ de pacientes (1).

Esta relativa baja sensibilidad de la citología ha ampliado la búsqueda de marcadores en el LP que ofrezcan un diagnóstico incruento de malignidad. La cuantificación del contenido de ADN mediante citometría de flujo tiene una sensibilidad similar a la citología (34). La mayoría de las neoplasias son aneuploides (niveles anormales de ADN), en contraposición a los DP benignos que suelen ser diploides (niveles normales de ADN), pero existe un solapamiento significativo (35). El empleo de marcadores tumorales en el LP también ha ofrecido resultados dispares, dependiendo del tipo de marcador, punto de corte seleccionado, método empleado para su determinación, tipo histológico de tumor o población estudiada. Ningún marcador de forma aislada parece lo suficientemente sensible y sólo la combinación de diversos marcadores tumorales, entre los que probablemente se debería incluir el antígeno carcinoembrionario (CEA) y quizá el CIFRA 21-1, puede incrementar el rendimiento diagnóstico $(36,37)$. Una excepción podría ser, si se confirman los resultados iniciales prometedores (38), la detección de la actividad de telomerasa en LP por una técnica de PCR-ELISA. En un estudio se detectó actividad de telomerasa en el 91,4\% de 70 DP malignos, en el 5,8\% de 52 DP no malignos y, lo que es más interesante, en el 90,9\% de 22 DP muy sospechosos de malignidad pero con citología o histología negativas (38).
OPCIONES CUANDO NO SE OBTIENE UN DIAGNÓSTICO CON LA TORACOCENTESIS INICIAL

Cuando no se obtiene un diagnóstico después de una toracocentesis inicial que incluya la determinación de ADA y una citología del LP, existen varias opciones. En primer lugar, es recomendable realizar una tomografía computadorizada (TC) espiral, con la que evaluar la posibilidad de una embolia pulmonar y la presencia de infiltrados pulmonares o adenopatías mediastínicas (39). Además, la demostración de nódulos pleurales o de engrosamientos pleurales nodulares es un dato prácticamente exclusivo del DP maligno (40). Si la TC espiral no demuestra embolia pulmonar, existen cinco opciones disponibles más: observación, broncoscopia, biopsia pleural, toracoscopia o toracotomía con biopsia abierta.

\section{OBSERVACIÓN}

Se trata de la mejor opción en el paciente que está mejorando y no tiene infiltrados pulmonares. Si el paciente tiene una neoplasia, probablemente no mejorará de forma espontánea. Por el contrario, la mayoría de los DP idiopáticos tienen un curso benigno, por lo que parece prudente adoptar una actitud conservadora (41). En estos casos no se recomienda tratamiento antituberculoso, independientemente del resultado de la prueba de la tuberculina, si los niveles de ADA pleural no están elevados. Se debe recordar que entre un 15 a $20 \%$ de los exudados pleurales quedan sin un diagnóstico definitivo.

La observación también puede ser una alternativa razonable a procedimientos más invasivos y costosos como la toracoscopia, cuando sospechamos un DP maligno y la citología pleural es negativa. Esta afirmación se fundamenta en la mortalidad elevada del DP maligno y en las opciones terapéuticas limitadas para el mismo.

\section{BRONCOSCOPIA}

La broncoscopia es útil en el diagnóstico del DP sólo si se cumple alguna de las siguientes condiciones (42): a) existen infiltrados pulmonares en la radiografía o TC torácicas; b) el paciente tiene hemoptisis, lo cual es muy sugestivo de lesión endobronquial; y c) el DP es masivo pero no desplaza el mediastino contralateralmente.

\section{BIOPSIA PLEURAL CON AGUJA}

La biopsia pleural con aguja sirve para diagnosticar tuberculosis pleural y, en menor medida, malignidad. Por lo que hace referencia a la primera, ya se ha comentado la disponibilidad actual de marcadores muy eficaces en el LP para establecer el diagnóstico. En los últimos años, la emergencia de tuberculosis multirresistente ha hecho que los cultivos tengan una importancia crucial para efectuar estudios de sensibilidad farmacológica que guíen el tratamiento. Por eso, algunos consideran que se debe realizar una biopsia pleural cuando se sospecha tuberculosis con el fin de cultivar la pieza. No obstante, sólo un tercio de los pacientes con pleuritis tuberculosa tendrán cultivo positivo de la biopsia pleural y negativo del LP (43). Además, hasta el momento no se ha descrito ningún 
paciente que haya desarrollado una tuberculosis multirresistente diseminada después de presentar un DP que se haya tratado con la pauta estándar de antituberculosos. Por todo ello, en nuestro medio rara vez está indicada la biopsia pleural para diagnosticar una pleuritis tuberculosa.

La biopsia pleural tiene menor sensibilidad que la citología para diagnosticar malignidad (aproximadamente 45\%). Esta limitación se debe a varios factores (44) que incluyen el estadio de la enfermedad en el momento de la biopsia, la mayor frecuencia de invasión maligna de la pleura visceral en contraposición a la parietal accesible a la biopsia, la naturaleza focal de la neoplasia y los DP que no están directamente producidos por la invasión maligna pleural (DP paramalignos). Un estudio mostró que sólo el 7,1\% de 281 pacientes con DP maligno se diagnosticaron con biopsia pleural cuando la citología era negativa (45).

\section{TORACOSCOPIA}

La toracoscopia médica, que se realiza bajo anestesia local y sedación, tiene una rentabilidad diagnóstica del 90 al 100\% en el DP maligno (46). Los pocos falsos negativos suelen ser casos de mesotelioma maligno en fase inicial. Cuando se sospecha una etiología neoplásica y la citología del LP es negativa, es preferible realizar una toracoscopia que una biopsia pleural con aguja, ya que diagnosticará malignidad en más del $90 \%$ de estos pacientes. Además, la toracoscopia tiene una vertiente terapéutica, al permitir realizar una pleurodesis con talco en los DP malignos. Por el contrario, la toracoscopia no tiene ventajas sobre la biopsia pleural para diagnosticar pleuritis tuberculosa.

\section{MANEJO DE LAS CAUSAS MÁS FRECUENTES DE DERRAME PLEURAL}

\section{INSUFICIENCIA CARDÍACA}

La insuficiencia cardíaca es la causa más frecuente de DP y, en particular, de trasudado. Dos terceras partes de los pacientes con insuficiencia cardíaca congestiva tienen DP, mayoritariamente bilateral. Se debe realizar una toracocentesis siempre que la presentación clínica sea atípica, como en las siguientes circunstancias (47): DP unilateral, DP bilateral con gran diferencia de tamaño entre un lado y otro, DP bilateral sin cardiomegalia, presencia de fiebre o dolor torácico y persistencia del DP a pesar del tratamiento diurético. En la insuficiencia cardíaca, el LP es un trasudado y, aunque la concentración de proteínas, LDH y colesterol pleurales aumenta significativamente con el tratamiento diurético, pocas veces se alcanzan los valores propios de un exudado (48).

El tratamiento es el común de la insuficiencia cardíaca (diuréticos, inhibidores de la enzima conversora de la angiotensina, digoxina), aunque ocasionalmente puede ser beneficioso evacuar volúmenes de 0,5 a 1,5 litros de LP mediante toracocentesis para aliviar con más rapidez la disnea intensa de pacientes con DP cuantiosos.

\section{DERRAME PLEURAL PARANEUMÓNICO}

Cualquier DP que se asocie con neumonía bacteriana, absceso pulmonar o bronquiectasias es un DPP. Los DPP que requie- ren un tubo de toracostomía para su resolución se denominan complicados. El empiema es la presencia de pus en el espacio pleural y representa el estadio final de un DPP complicado.

Se debe realizar una toracocentesis inmediata en todo paciente con neumonía y DP asociado, salvo que aquélla tuviera riesgos por el pequeño tamaño del derrame. Si se extrae pus, enviaremos la muestra para Gram y cultivo y se colocará con carácter urgente un tubo de drenaje torácico, preferiblemente de grueso calibre ( $\geq 24 \mathrm{~F})$. Si el LP no es purulento se analizarán, además de los parámetros microbiológicos (Gram, cultivo), las concentraciones de $\mathrm{pH}$, glucosa y LDH. Se acepta como indicación de tubo de toracostomía la presencia de un Gram o cultivo positivos, un $\mathrm{pH}<7$ o una glucosa $<40 \mathrm{mg} / \mathrm{dl}$. No existe acuerdo sobre la necesidad inmediata de drenaje pleural cuando el $\mathrm{pH}$ está entre 7 y 7,2, la glucosa entre 40 y $60 \mathrm{mg} / \mathrm{dl}$ o la LDH pleural es mayor que 3 veces el límite superior de la LDH sérica. En estos casos, que Light clasifica como DPP complicados limítrofes o borderline (49), nos inclinaremos por el drenaje si el paciente cumple alguna de las siguientes condiciones: DP que ocupa más de la mitad del hemitórax o con loculaciones, aspecto clínico de gravedad, fiebre y leucocitosis persistente a pesar del tratamiento antibiótico, edad avanzada, comorbilidad o determinados gérmenes responsables de la neumonía (anaerobios, bacterias piógenas, $K$. pneumoniae). La existencia de un nivel hidroaéreo intrapleural en la radiografía de tórax es también una indicación absoluta de drenaje (50). Es preferible utilizar un tubo de toracostomía de pequeño calibre (8-14F) en los DPP complicados no purulentos (51). Probablemente, es recomendable realizar una toracocentesis terapéutica, además de diagnóstica, tan pronto como se reconozca un DPP (49), optando con posterioridad por el tubo de drenaje si el LP no purulento se locula o bien se reacumula y tenía, en la primera toracocentesis, características indicativas de una posible mala evolución (Gram o cultivo positivo, $\mathrm{pH}<7$, glucosa $<40 \mathrm{mg} / \mathrm{dl}$, $\mathrm{LDH}>1000 \mathrm{UI} / \mathrm{l})$.

La loculación del LP indica un alto nivel de inflamación en el espacio pleural. La mayoría de los DPP loculados requieren drenaje. En los pacientes con DPP loculado que tienen cualquiera de los factores microbiológicos o bioquímicos de mala evolución ya mencionados, se intentará romper las loculaciones para obtener un drenaje completo de la cavidad pleural. Esto se consigue instilando fibrinolíticos a través del tubo de toracostomía (p. ej. $100.000 \mathrm{U}$ de urocinasa disueltas en $100 \mathrm{ml}$ de suero fisiológico cada día) o realizando una toracoscopia, un procedimiento que también se indicará si fallan los fibrinolíticos. La decorticación se reserva para el pulmón atrapado.

\section{DERRAME PLEURAL MALIGNO}

El cáncer de pulmón en el hombre y el cáncer de mama en la mujer son las principales causas de DP maligno. Cuando un DP es secundario a metástasis pleurales se debe plantear la posibilidad de obliterar el espacio pleural mediante pleurodesis. Los pacientes que se sometan a este procedimiento deben cumplir varios requisitos. En primer lugar, el paciente no tendrá un mal estado general o una expectativa de vida muy corta (menos de 1 mes), ya que en estos casos es preferible optar por las toracocentesis terapéuticas periódicas (44). En segundo lugar, la calidad de vida del paciente debe estar limitada por la disnea. En tercer lugar, una toracocentesis terapéutica 
debe mejorar ostensiblemente la disnea. Finalmente, el pulmón debe estar completamente reexpandido para conseguir una sínfisis pleural exitosa (52).

La pleurodesis química consiste en la inyección de un agente irritante que provoca una reacción pleural intensa capaz de fusionar las pleuras visceral y parietal. Aunque no existe un agente esclerosante ideal, en la actualidad el más utilizado es el talco administrado bien en suspensión salina (slurry) a través del tubo de toracostomía o por insuflación durante una toracoscopia (53). Aunque raras veces (1\%), se han descrito complicaciones graves como distrés respiratorio del adulto que parecen relacionadas con el tamaño de las partículas de talco (54). Por ello, para algunos autores es preferible el empleo de alternativas como las tetraciclinas o derivados (55). La técnica de pleurodesis es sencilla (56). Después de que el tubo de toracostomía haya evacuado completamente el líquido del espacio pleural y el pulmón esté reexpandido, se instilan $5 \mathrm{~g}$ de talco o $500 \mathrm{mg}$ de doxiciclina disueltos en 50-100 $\mathrm{ml}$ de suero salino a través del tubo. Inmediatamente después se cierra el tubo durante una a dos horas, sin necesidad de que el paciente efectúe cambios de posición durante este período. Con posterioridad se despinza el tubo y se aplica presión negativa $(20 \mathrm{~cm} \mathrm{H20)}$, retirándolo en las primeras 72 horas si el pulmón permanece reexpandido y se ha reducido el débito de LP ( $<100-150 \mathrm{ml}$ en 24 horas). Se consigue una pleurodesis eficaz en más de dos terceras partes de los pacientes.

El shunt pleuroperitoneal es una medida paliativa eficaz en pacientes con DP maligno cuantioso y pulmón atrapado (contraindicación para la pleurodesis). El shunt, que se coloca bajo anestesia local, consiste en dos catéteres conectados por una cámara que actúa de bomba y que contiene dos válvulas unidireccionales. El líquido fluye del espacio pleural a la cámara y de ésta a la cavidad peritoneal, después de que el paciente bombee diariamente sobre la cámara. Aunque su eficacia es del $95 \%$, en aproximadamente el $15 \%$ de sujetos se requiere su revisión o substitución por oclusión o infección (57).

\section{Bibliografía}

1. Light RW. Pleural diseases. $4^{\mathrm{a}}$ ed. Philadelphia: Lippincott Williams \& Wilkins, 2001

2. Light RW, MacGregor MI, Luchsinger PC, Ball WC. Pleural effusions: the diagnostic separation of transudates and exudates. Ann Intern Med 1972; 77: 507-13

3. Vives M, Porcel JM, Vicente de Vera MC, Ribelles E, Rubio M. A study of Light's criteria and possible modifications for distinguishing exudative from transudative pleural effusions. Chest 1996; 109: 1503-7.

4. Porcel JM, Vives M. Classic, abbreviated, and modified Light's criteria The end of the story?. Chest 1999; 116: 1833-4.

5. Porcel JM, Vives M, Vicente de Vera MC, Cao G, Rubio M, Rivas MC. Useful tests on pleural fluid that distinguish transudates from exudates. Ann Clin Biochem 2001; 38: 671-675.

6. Joseph J, Badrinath P, Basran GS, Sahn SA. Is the pleural fluid transudate or exudate? A revisit of the diagnostic criteria. Thorax 2001; 56: $867-0$.

7. Burgess LJ, Maritz FJ, Taljaard JJF. Comparative analysis of the biochemical parameters used to distinguish between pleural transudates and exudates. Chest 1995; 107: 1604-9.

8. Romero-Candeira S, Fernández C, Martín C, Sánchez-Paya J, Hernández L. Influence of diuretics on the concentration of proteins and other components of pleural transudates in patients with heart failure. Am J Med 2001; 110: 681-6.

9. Heffner JE, Brown LK, Barbieri CA. Diagnostic value of tests that discriminate between exudative and transdutive pleural effusions. Chest 1997; 111: 970-80

10. Romero S, Martínez A, Hernández L et al. Light's criteria revisited: consistency and comparison with new proposed alternative criteria for separating pleural transudates from exudates. Respiration 2000; 67: 18-23.

11. Ansari T, Idell S. Management of undiagnosed persistent pleural effusions. Clin Chest Med 1998; 19: 407-17.

12. Scheurich JW, Keuer SP, Graham DY. Pleural effusion: comparison of clinical judgment and Light's criteria in determining the cause. South Med J 1989; 82: 1487-91.

13. Porcel JM, Alvarez M, Salud A, Vives M. Should a cytologic study be ordered in transudative pleural effusions? Chest 1999; 116: 1836-1837.

14. Alemán C, Alegre J, Armadans L et al. The value of chest roentgenography in the diagnosis of pneumothorax after thoracentesis. Am J Med 1999; 107: 340-3.

15. Romero S. Nontraumatic chylothorax. Curr Opin Pulm Med 2000; 6: 287-91.

16. Martínez-García MA, Cases-Viedma E, Cordero-Rodríguez PJ et al Diagnostic utility of eosinophils in the pleural fluid. Eur Respir J 1999; 15: $166-9$.
17. Blavia-Aloy R, Rodriguez-Sanchón B, Manresa-Presas F. Derrame pleural eosinofílico: estudio de 25 casos y revisión de la literatura. An Med Interna (Madrid) 1988; 5: 9-13.

18. Sarodia BD, Goldstein LS, Laskowski DM, Mehta AC, Arroliga AC. Does pleural fluid $\mathrm{pH}$ change significantly at room temperature during the first hour following thoracentesis? Chest 2000; 117: 1043-1048.

19. Jiménez-Castro D, Díaz G, Pérez-Rodríguez E, Prieto D, Yusen RD. Modification of pleural fluid $\mathrm{pH}$ by local anesthesia. Chest 1999; 116: 399-402.

20. Valdés L, Álvarez D, San José E et al Tuberculous pleurisy: A study of 254 patients. Arch Intern Med 1998; 158: 2017-21

21. Ocaña I, Martínez-Vázquez JM, Segura RM, Fernández de Sevilla T, Capdevila JA. Adenosine deaminase in pleural fluids: test for diagnosis of tuberculous pleural effusion. Chest 1983; 84:51-53.

22. Lee YCG, Rogers JT, Rodríguez RM et al. Adenosine deaminase in nontuberculous lymphocytic pleural effusions. Chest 2001; 120: 35661.

23. Porcel JM, Vives M. Adenosine deaminase in nontuberculous lymphocytic pleural effusions. Chest (en prensa).

24. Pérez-Rodríguez E, Pérez-Walton IJ, Sánchez-Hernández JJ, et al. ADA1/ADAp ratio in pleural tuberculosis: an excellent diagnostic parameter in pleural fluid. Respir Med 1999; 93: 816-21.

25. Light RW, Ferrer J. Diagnóstico de la pleuritis tuberculosa. Arch Bronconeumol 1999; 35: 105-7.

26. Villena V, López-Encuentra A, Echave-Sustaeta J, Martín-Escribano P, Ortuño-de-Solo B, Estenoz-Alfaro J. Interferon-g in 388 immunocompromised and immunocompetent patients for diagnosing pleural tuberculosis. Eur Respir J 1996; 9: 2635-9.

27. Wongtim S, Silachamroon U, Ruxrungtham K et al. Interferon gamma for diagnosing tuberculous pleural effusions. Thorax 1999; 54: 921-4

28. Querol JM, Mínguez J, García-Sánchez E, Farga MA, Gimeno C, García-de-Lomas J. Rapid diagnosis of pleural tuberculosis by polymerase chain reaction. Am J Respir Crit Care Med 1995; 152: 1977-81.

29. Villena V, Rebollo MJ, Aguado JM, Galán A, López Encuentra A, Palenque E. Polymerase chain reaction for the diagnosis of pleural tuberculosis in immunocompromised and immunocompetent patient. Clin Infect Dis 1998; 26: 212-4.

30. Villegas MV, Labrada LA, Saravia NG. Evaluation of polymerase chain reaction, adenosine deaminase, and interferon-g in pleural fluid for the diferential diagnosis of pleural tuberculosis. Chest 2000; 118: 1355-64.

31. Porcel JM, Vives M, Gázquez I, Vicente de Vera MC, Pérez B, Rubio $M$. Usefulness of pleural complement activation products in differentiating tuberculous and malignant effusions. Int $\mathrm{J}$ Tuberc Lung Dis 2000; 4: 76-82. 
32. Porcel JM, Gázquez I, Vives M, Pérez B, Rubio M, Rivas MC. Diagnosis of tuberculous pleuritis by the measurement of soluble interleukin 2 receptor in pleural fluid. Int J Tuberc Lung Dis 2000; 4: 975-9.

33. Light RW. Diagnostic principles in pleural diseases. Eur Respir J 1997; 10: 476-81.

34. Saha I, Dey P, Vhora H, Nijhawan R. Role of DNA flow cytometry and image cytometry on effusion fluid. Diagn Cytopathol 2000; 22: 81-5.

35. Rodriguez de Castro F, Molero T, Acosta O et al. Value of DNA analy sis in addition to cytological testing in the diagnosis of malignant pleural effusions. Thorax 1994; 49: 692-4.

36. Ferrer J, Villarino MA, Encabo G et al. Diagnostic utility of CYFRA 21-1, carcinoembryonic antigen, CA 125, neuron specific enolase, and squamous cell antigen level determinations in the serum and pleural fluid of patients with pleural effusions. Cancer 1999; 86: 1488-95.

37. Ferrer-Sancho J. Marcadores tumorales en líquido pleural. Arch Bronconeumol 2000; 6: 295-297.

38. Yang CT, Lee MH, Lan RS, Chen JK. Telomerase activity in pleural effusions: diagnostic significance. J Clin Oncol 1988; 16: 567-73.

39. Bates SM, Ginsberg JS. Helical computed tomography and the diagnosis of pulmonary embolism. Ann Intern Med 2000; 132: 240-2.

40. Arenas-Jiménez J, Alonso-Charterina S, Sánchez-Paya J, FernándezLatorre F, Gil-Sánchez S, Lloret-Llorens M. Evaluation of CT findings for diagnosis of pleural effusions. Eur Radiol 2000; 10: 681-90.

41. Ferrer JS, Muñoz XG, Orriols RM, Light RW, Morell FB. Evolution of idiopathic pleural effusion. A prospective, long-term follow-up study. Chest 1996; 109: 1508-13.

42. Jurado B, Sánchez L, Sánchez R, García FL, Cosano A, Muñoz L. Utilidad de la broncofibroscopia en el estudio del derrame pleural. An Med Inter (Madrid) 1995; 12: 225-8.

43. Light RW. Closed needle biopsy of the pleura is a valuable diagnostic procedure. Con closed needle biopsy. J Bronchol 1998; 5: 332-6.
44. Antony VB, Loddenkemper R, Astoul P et al. Management of malignant pleural effusions. Am J Respir Crit Care Med 2000; 162: 19872001.

45. Prakash UB, Reiman HM. Comparison of needle biopsy with cytologic analysis for the evaluation of pleural effusion: analysis of 414 cases. Mayo Clin Proc 1985; 60: 158-164.

46. Colt HG. Thoracoscopy. Window to the pleural space. Chest 1999; 116 : 1409-15.

47. Kinasewitz GT. Transudative effusions. Eur Respir J 1997; 10: 714-8.

48. Shinto RA, Light RW. Effects of diuresis on the characteristics of pleural fluid in patients with congestive heart failure. Am J Med 1990; 88: 230-4.

49. Light RW, Porcel JM. Derrame pleural paraneumónico y empiema. Med Clin (Barc) 2000; 115: 384-91.

50. Heffner JE. Indications for draining a parapneumonic effusion: an evidence-based approach. Semin Respir Infect 1999; 14: 48-58.

51. Porcel JM, Rodriguez-Panadero F. ¿Cuándo y cómo drenar un derrame pleural?. Med Clin (Barc) (en prensa).

52. Antunes G, Neville E. Management of malignant pleural effusions. Thorax 2000; 55: 981-3.

53. Sahn SA. Talc should be used for pleurodesis. Am J Respir Crit Care Med 2000; 162: 2023-4.

54. Ferrer J, Villarino MA, Tura JM, Traveria A, Light RW. Talc preparations used for pleurodesis vary markedly from one preparation to another. Chest 2001; 119:1901-5.

55. Light RW. Talc should not be used for pleurodesis. Am J Respir Crit Care Med 2000; 162: 2024-2026.

56. Colt HG, Mathur PN. Manual of pleural procedures. Philadelphia: Lippincott Williams \& Wilkins; 1999; 155-61.

57. Genc O, Petrou M, Ladas G, Goldstraw P. The long-term morbidity of pleuroperitoneal shunts in the management of recurrent malignant effusions. Eur J Cardiothorac Surg 2000; 18: 143-6. 


\section{OCIEDADESPANOIA MEDICINAINTERNA

\section{pr ograma cien tífico}

\section{martes 5}

ENTREGA DE DOCUMENTACIÓN

TALLERES DE FORMACIÓN CONTINUADA "Tratamiento de la fi brilación auriaular paroxística yorónica"

Moderador: Dr. Luis) iménez deD iego (H . Clínico San Carlos Madrid)

"La informática en la mejora dela atención sanitaria de medi dina interna en el sigloXXI"

Dr. Migue Ángel Mateos H ernández ( $\mathrm{H}$. Fundación Alcorcón)

Dr. J avier M ateos H ernández ( $\mathrm{H}$. General U niversitario. Guadalajara)

17.30-18.30 MESA REDONDA (1)

"Gestión dínica en Medicina I nterna"

M oderador: Dr. J osé A ntonio Santos Calderón (H. M onte San I sidoro. León)

Getión dínica. A spectos conceptuales

Getión dínica en Medicina Interna

Gestión dínica y cali dad asistencial

17.30-18.30 MESA REDONDA (2)

"Bi oćica al final dela vida"

M oderador: Dr. Eloy Pacho Jiménez (Fundación Jiménez Díaz. Madrid)

Ética del cuidado: cuando no es posiblecurar

Malasnoticias ¿informar ocallar?

Voluntades antici padas testamento vital

BIENVENIDA. SESIÓN CONMEM ORATIVA DEL 50 ANIVERSARIO DE LA FUNDACIÓN SEMI

20.00 COLOCACIÓN DE POSTERS PARA LA PRÓXIMA JORNADA

\section{miércoles 6}

08.30-09.15 VISITA DE PÓSTER (1)

$08.30-09.15$

REUNIONES CON EL EXPERTO (1 y 2)

"A vancesen $\mathrm{A}$ SI DA"

D r. J osé Ma Peña Sánchez de R ivera (H . U . La Paz. Madrid)

Dr. J osé M a . Gatell (H. Clinici Provincial. Barcelona)

"Osteoporosis"

Dr. A dolfo Díez Pérez (H. del M ar. Barcelona)

Dr. Jesús González M acías (H . M arqués de Valdecilla.

Santander)
$11.30-13.00$

09.15-09.30 CEREMONIA DE INAUGURACIÓN OFICIAL

09.30-11.00 SESIÓN PLENARIA (1)

"A vancesen e SI DA"

M oderador: D r. José M. Gatell (H . Clinici Provincial. Barcelona)

A vancesen la inmunopatogénesisdel SI D A

Tratami ento antirretroviral: año 2002

A vancesen la prevención y tratamiento delasinfecciones oportunistas

11.30-13.00 MESASREDONDA (3)

"Osteoporos' $S$ "

Moderador. Dr. A dolfo Díez Pérez (H . del Mar. Barcelona)

La conexión entre la enfermedad disipémica y la osteopor osis

Tratami ento y prevenci ón de la osteopor os scorti coi dea

Osteoporosisdel varón

MESA REDONDA (4)

"N uevas etrategi as terapéuticas en enfermedades autoinmunes"

M oderador: $\mathrm{Dr}$. M elchor A lvárez de M ón

(H. U. Príncipe de A sturias. A lcalá de H enares)

Estrategi asde modulación funcional de sstema inmune

Reconstitución del sistema inmune Trasplante de precursores hematopoyéticos

Estrategi as de deplecci ón de cólulas inmunocompetentes

MESA REDONDA (5)

"I nvestigadión en A terosder osis"

Moderador: Dr. Manuel de Oya Otero (Fundación Jimé nez Díaz. Madrid)

El endotelio, factor protector o desencadenante en la patología cardi ovascular

Lipoproténa a: dela dínica a la genética

EI ratón, un nuevo aliado en el esdareci mi ento de la enfermedad cardi ovasaular

$13.15-14.00$

COMUNICACIONESORALES

$16.00-17.30$

CONFERENCIA MAGISTRAL

SESION PLEN ARIA (2)

"F actoresde riesgo vasaular"

Moderador: Dr. BlasGil Extremera (H . Clínico San C ecilio. Granada)

Disipemia

H i pertensión arterial

H omoci sténa 
17.30-19.00 MESA REDONDA (6)

"H ipertensión arterial"

M oderador: D r. Manue Luque Otero (H. ClínicoSan Carlos Madrid)

¿Cómo mejorar el mal control dela hi pertensión arterial?

¿Quénosaportará la genética para el cuidado de los pacientes?

¿Quécriteriosutilizar para elegir un anti hiperten $\mathrm{s}$ vo?

MESA REDONDA (7)

"A tendión a laspersonasmayoresen Medicina

Interna"

M oderador: Dr. Francisco A rnalich Fernández (H . U . La Paz. Madrid)

Valoracion de la gravedad y pronóstico en personas mayorescon comorbilidad y di scapaci dad

Prevención primaria y secundaria de la enfermedad cerebrovascular

Malnutrición y soporte nutricional

17.30-19.00 VISITA DE PÓSTER (2)

17.30-19.00 TALLER FORMACIÓN CONTINUADA (3)

"A plicación de métodos diagnósticos en Medicina I nterna"

Dr. Fernando Carballo Álvarez (H . General U niversitario. Guadalajara)

20.00

COLOCACIÓN DE PÓSTER PARA LA PRÓXIMA JORNADA

jueves 7

\subsection{0-09.15 VISITA DE PÓSTER (1)}

08.30-09.15 REUNIÓN CON EL EXPERTO (3 y 4)

"Enfermedadesautoi nmunes"

Dr. José Font Franco (H. Clinic i Provincial. Barcelona)

Dr. Antonio Gil A guado (H . U . La Paz. Madrid)

"E cocardi ografía: Todo lo que debe saber el interni sta"

Dr. Miguel Ángel García Fernández (H .G.U. Gregorio

Marañón. Madrid)

09.30-11.00 SESIÓN PLENARIA (3)

"D esarrollo y perspectivasde la Medicina I nterna"

M oderador: Dr. Miguel Vilardell Tarrés ( $H$. Vall

d’H ebrón. Barcelona)

Medicina Interna en Europa

Si tuación actual dela Medicina Interna

Futuro dela Medicina Interna

11.30-13.00 MESA REDONDA (8)

"DM tipo 2 yśndrome plu rimetabólico"

Moderador: Dr. Manuel Serrano Ríos (H. Clínico San Carlos Madrid)

Diabetes tipo 2, insulinorresistencia y riesgo cardi ovascular: de la epi demi ología a la eti opatogenia Prevención de enfermedad cardiovasaular en Diabetes tipo 2

N uevos fármacos anti di a béti cos y ri esgo cardi ovascular en diabetes

MESA REDONDA (9)

"Asma y enfermedadesinmundógicas del pulmón"

Moderador: Dr. J osé L uis Á Ivarez-Sala Walther

(H. Clínico San Carlos Madrid)
N uevos tratamientosen el asma

Enfermedad pulmonar intersticial difusa: basesactuales desu dasificación

Enfoque diagnóstico de la enfermedad pulmonar intersticial difusa

MESA REDONDA (10)

"I n fecci on es cardi ova sculares' di agnósti co y tratami ento"

Moderador: Dr. Emilio Bouza (H. G. U . Gregorio Marañón. Madrid)

Endocarditissobreválvula nativa y protésica

N uevasperspectivas di agnósti casy terapéuticasde la endo carditisinfeccios

I nfección decatéter y prótesisendovasculares

11.30-13.00 COMUNICACIONESORALES

13.15-14.00 CONFERENCIA MAGISTRAL

"H iperinsulismoy susconseauendasdínicas"

Dr. E. Ferranini (Universidad dePisa)

16.00-17.30 SESION PLENARIA (4)

"I nsufi ciencia car díaca: dela evidenda

centífica a la práctica dínica diaria"

M oderador: Dr. Pedro C onthe Gutiérrez (H . G. U . Gregorio Marañón. Madrid)

La I.C . en Europa: magnitud del problema

Posibilidadesy limitaciones de la ecocardiografía en e diagnóstico de la I.C.

Últimasaportacionesterapéuticasen e tratamiento farmacológico de la I.C.

\subsection{0-19.00 MESA REDONDA (11)}

"G astropr otección"

Moderador: Dr. Manuel Díaz-R ubio (H . Clínico San

Carlos Madrid)

Gastroprotección en pacientescon A I NES:

en qué pacientesy cómo

EI uso de inhibi dores de la COX-2: ventajase indicaciones Profilaxis de la gastropatía en pacientes en situaciones deestrés

MESA REDONDA (12)

"A vancesen la tuberculosis"

Moderador: Dr. José M. Aguado García (H. U. 12 de Octubre. Madrid)

N uevos aspectos epi demi ológi cos y patogéni cos de la tuberculosis susimplicacionespara ed dínico

A vances en el diagnósti co de la infección y de la enfermedad tuberculosa

Profilaxisy tratamiento de la tuberculosis. las opor tun idades perdi das

17.30-19.00 VISITA DE PÓSTER (2)

17.30-19.00 TALLER FORMACIÓN CONTINUADA (4 y 5) "Tratamiento de dolor"

Dr. Joaquín Insausti Valdivia ( $H$. Severo Ochoa. Leganés)

"Tratamiento de ictus"

Dr. J. MatíasGuiú (H. General. A licante)

Dr. Antonio Gil N uñez (H. G. U. Gregorio Marañón. Madrid)

18.00-19.00 ASAM BLEA SEM I

19.00 COLOCACIÓN DE PÓSTER PARA LA PRÓXIMA JORNADA 


\section{viernes 8}

08.30-09.15 VISITA DE PÓSTER (1)

08.30- 09.15 REUNIÓN CON EL EXPERTO (5 y 6) "D iabetes ti po 2 y ś drome plu ri metabóli co" Dr. LuisFelipe Pallardo (H. U. La Paz. Madrid) Dr. Santiago Durán García (H . U . N uestra Sra. de ValmeSevilla)

"I nfección nosocomial"

Dr. J osé Luis Pérez A rellano (H . Insular. Las Palmas)

09.30-11.00 SESIÓN PLENARIA (5)

"N u evas per spectivas en el LES y ś ndrome anti fosfolí pi do"

Moderador: Dr. J osé Font Franco (H. Clinic i Provincial. Barcelona)

A vancesen la patogenia del Síndrome A ntifơfolípi do

A ctualidad de los marcadores bi ológi cos de acti vidad en d LES

Perspectivasterapéuticasen el LES

11.30-13.00 MESA REDONDA (13)

"Lasnuevasinsulinas aplicadiones dínicas"

Moderador: Dr. Juan J. Vázquez R odríguez (H. U. La Paz. Madrid)

Lasinsulinasdel sigloXXI

A nálogos de la insulina en la diabetestipo 1

A nálogos de la insulina en la diabetestipo 2

MESA REDONDA (14)

"A vancesen H epatitisvírica"

D r. J aume Guardia M asó (H . Vall d’H ebrón. Barcelona)

E stado actual y perspectivas en el tratamiento de la hepatitis C

Estado actual y perspectivas en el tratami ento de la hepatitis B

H epati ti sviral en el hígado trasplantado

MESA REDONDA (15)

"I nfecci ón en el inmigrante"

M oderador: Dr. J osé Luis Pérez A rellano (H . Insular. Las Palmas)

¿A qué llamamos neumonía nosocomial y cómo la tratamos?
A vances en la fisiopatología y tratamiento del shock śptico

Sepsis de origen urinario

11.30-13.00 COMUNICACIONES ORALES

13.15-14.00 CONFERENCIA M AGISTRAL

"A plicaciones diagnósticas de la tomografía de emisión depositrones"

Dr. José Luis Carreras Delgado (H . Clínico San Carlos Madrid)

16.00-17.30 SESION PLENARIA (6)

"Enfermedad tromboembólica venosa"

M oderador: Dr. M anuel Monreal Bosch (H . GermansTríasi Pujol. Badalona)

Enfermedad tromboembólica y cáncer

Genética y enfermedad tromboembólica

Situación actual del registro de enfermedad tromboembólica de la SEMI

17.30-19.00 MESA REDONDA (16)

"H epati tisno víricas"

M oderador: Dr. Jorge Quiroga Vila (Clínica U niversitaria de $\mathrm{N}$ avarra. Pamplona)

\section{H epati tisalcohólica}

H epatitisautoi nmune

Estatohepati ti sno alcohólica

MESA RED ONDA (17)

"Actualización en Demencias"

Moderador: Dr. Félix Bermejo (H. U. 12 de Octubre. Madrid)

Guías de práctica dínica en demencias

Demencia ti po Alzhei mer

Demencia no-A lzhei mer

17.30-19.00 VISITA DE PÓSTER (2)

17.30-19.00 TALLER FORMACIÓN CONTINUADA

"Calidad asistencial en insufi iencia cardíaca"

Dr. R amón Pujol Farriols (C.U . de Bellvitge. H ospitalet de Llobregat)

\section{Sec r et aría Cien tífica}

H ospital U niversitario La Paz Servicio de M edicina I nterna Paseo de la Castellana, 26128046 M adrid Telf. y Fax: 917277482 e-mail: francisco.arnalich@uam.es
Sed e

Palacio M unicipal de Congresos de M adrid Campo de las $\mathrm{N}$ aciones

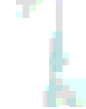

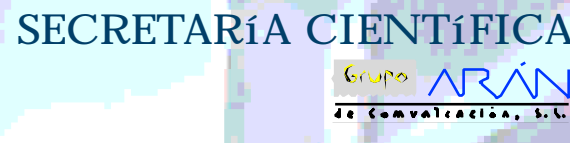

Castelló, 128-1. 28006 M adrid. Tel. 9178200 33. Fax. 915615787 M untaner, 270-4A. 08021 Barcelona. Fax: 932017028

e-mail: congreso@grupoaran.com http:/ / www.medicinainterna2002.org 


\section{AIVALES IE MEDICINA INTERNA + Seminarios DE A.M.I.}

LA REVISTA DE MEDICINA INTERNA

(14 núms. año)

$\square$ MIR y Estudiantes*:

$\square$ Organismos y Empresas:

*Los MIR y Estudiantes deberán adjuntar documento acreditativo

\section{BOLETÍN DE SUSCRIPCIÓN AÑO 2002}

\section{DIRECCIÓN DE ENVÍO}

Nombre y apellidos

Dirección

Población Cod. Postal Provin.

e-mail

Especialidad Centro Cargo

SUSCRÍBANME A:

\section{ANALES DE MEDICINA INTERNA}

$\square \quad$ A través de mi cuenta bancaria (cumplimento autorización adjunta)

$\square \quad$ Mediante talón $\mathrm{n} . \stackrel{\circ}{ }$ que adjunto

$\square \quad$ Contra reembolso

ORDEN DE PAGO POR DOMICILIACION BANCARIA

ANALES DE MEDICINA INTERNA

BANCO/CAJA

DIRECCIÓN POBLACIÓN C.P.

TITULAR DE LA CUENTA

CÓDIGO C/C.: BANCO SUCURSAL D.C N.․ CUENTA

Ruego a ustedes se sirvan tomar nota de que, hasta nuevo aviso, deberán adeudar en mi cuenta con esa entidad el recibo o letra que anualmente y a mi nombre les sean presentados para su cobro por

Les saluda atentamente,

(Firma)

de

de 20

\section{Más información o envíos a:}

